\title{
A study of the mechanism of IncRNA- CR594175 in regulating proliferation and invasion of hepatocellular carcinoma cells in vivo and in vitro
}

Quan Liu ${ }^{1+}$, Xuxu Yu ${ }^{2 \dagger}$, Minjie Yang ${ }^{2}$, Xiangke Li ${ }^{2}$, Xuejia Zhai ${ }^{2}$, Yujin Lian², Zhong Chen², Qingxia Fan², Lijie Song ${ }^{2^{*}}$ (i) and Wencai Li ${ }^{3^{*}}$

\begin{abstract}
Background: Hepatocellular carcinoma (HCC) is one of the cancers of highest incidence and mortality worldwide. The proliferation and invasion of tumor cells are the main reason for poor prognosis after HCC surgery. Long noncoding RNA (InCRNA) has been shown to play a key role in the progression of HCC. LncRNA-CR594175 is one of the highly expressed IncRNAs in HCC tumors and their metastatic tumors that we have obtained by the Highthroughput screening method.

Methods: To elucidate the role of IncRNA-CR594175 in regulating the proliferation and invasion of human hepatoma cell line, HepG2, we operated through IncRNA-CR594175 silencing to inhibit the progression of HCC, either through in vitro or in vivo experiments.

Results: We found that InCRNA-CR594175 was lower in adjacent non-cancerous tissues than in primary HCC, and was lower in primary HCC than in its metastasis. Silencing of IncRNA-CR594175 inhibited the proliferation and invasion of HepG2 cells and growth of subcutaneous tumors. The results revealed that IncRNA-CR594175, as a RNA sponge, broke the negative regulation of hsa-miR-142-3p on Catenin, beta-1 (CTNNB1), and once IncRNA-CR594175 was silenced, the hsa-miR142-3p regained its negative regulation on CTNNB1 which can promote HCC progression by activating the wnt pathway.
\end{abstract}

Conclusions: Our present study demonstrated for the first time that IncRNA-CR594175 silencing suppressed proliferation and invasion of HCC cells in vivo and in vitro by restoring the negative regulation of hsa-miR-142-3p on CTNNB1, laying a solid theoretical base for using IncRNA-CR594175 as genetic target therapy for HCC and offering a reasonable explanation for inactivation of miRNA in different tumors or in the tumor at different stages.

Keywords: IncRNA, IncRNA-CR594175, Hepatocellular carcinoma, Hsa-miR142-3p, HepG2

\footnotetext{
* Correspondence: culleen@163.com; liwencaipatho@126.com

${ }^{\dagger}$ Quan Liu and Xuxu Yu contributed equally to this work and are co-first authors.

${ }^{2}$ Department of Oncology, The First Affiliated Hospital of Zhengzhou University, Zhengzhou 450052, China

${ }^{3}$ Department of Pathology, The First Affiliated Hospital of Zhengzhou University, Zhengzhou 450052, China

Full list of author information is available at the end of the article
}

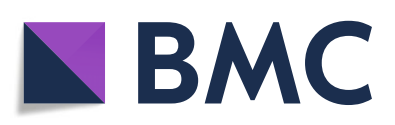

() The Author(s). 2020 Open Access This article is licensed under a Creative Commons Attribution 4.0 International License, which permits use, sharing, adaptation, distribution and reproduction in any medium or format, as long as you give appropriate credit to the original author(s) and the source, provide a link to the Creative Commons licence, and indicate if changes were made. The images or other third party material in this article are included in the article's Creative Commons licence, unless indicated otherwise in a credit line to the material. If material is not included in the article's Creative Commons licence and your intended use is not permitted by statutory regulation or exceeds the permitted use, you will need to obtain permission directly from the copyright holder. To view a copy of this licence, visit http://creativecommons.org/licenses/by/4.0/. The Creative Commons Public Domain Dedication waiver (http://creativecommons.org/publicdomain/zero/1.0/) applies to the data made available in this article, unless otherwise stated in a credit line to the data. 


\section{Background}

Hepatocellular carcinoma (HCC) is one of the cancers of highest incidence and mortality worldwide [1], featuring a low complete resection rate and a high postoperative recurrence rate $[2,3]$, which is mainly driven by high invasiveness and intrahepatic and/or extrahepatic metastasis [4]. Therefore, the study of the mechanisms involved in HCC cell proliferation and invasion may be of great significance to identify prognostic markers in HCC patients. At present, surgery combined with pre- and postoperative chemotherapy is the mainstay of treatment for HCC but this traditional therapeutic method doesn't work for postoperative recurrence and metastasis. So, in recent years, searching for new therapeutic targets for HCC treatment never stopped.

Both microRNAs (miRNAs) and lncRNAs play important roles in regulating cellular processes [5-7]. Going through all lncRNAs identified by the screening and sequencing of the transcriptome, lncRNA-CR594175 was the lncRNA most differentially expressed among adjacent non-cancerous tissues, $\mathrm{HCC}$ and metastases. The expression of lncRNA-CR594175 increases from adjacent non-cancerous, to primary HCC, to metastasis tissues, which suggests that IncRNA-CR594175 may be involved in proliferation and invasion of HCC. According to our screening data, CTNNB1 was highly correlated with the process of HCC development.

The pathogenesis of HCC is complex and Wnt/ CTNNB1 signaling pathway plays a central role in the hepatocarcinogenesis. According to medical basic research, Wnt/CTNNB1 signaling pathway can affect the process of HCC mainly by expression regulation of downstream genes and proteins. The increasing expression of CTNNB1 leads to cell proliferation according to the Wnt/CTNNB1 signaling pathway. It makes CTNNB1 a critical gene in the research of $\mathrm{HCC}$ regulation pathways. The inhibitory effects of Wnt signaling pathway by nonsteroidal anti-inflammatory drugs and valproic acid has been used as adjuvant for HCC therapy [8], therefore, CTNNB1 inhibitors become the new study direction in preventing precancerous lesions such as hepatitis and liver cirrhosis from deteriorating. It has already been verified that R-Etodolac, as the inhibitor of CTNNB1, can suppress proliferation of HCC cell lines HepG2 and Hep3B effectively [9]. In addition to this, a variety of miRNAs has been confirmed to have an influence on the process of $\mathrm{HCC}$ through the regulation of Wnt/ CTNNB1 signaling pathway [10-12] and the targets are different. It's uncommon for miRNA to involve Wnt signaling pathway targeting CTNNB1 until now, but our study revealed that miRNA hsa-miR-142-3p has a negative effect on the HCC progression by affecting the CTNNB1 pathway. The data indicated an increase in the expression level of hsa-miR142-3p from HCC metastasis to primary lesion and then to adjacent non-cancerous tissues, but it's puzzling that the expression of CTNNB1 protein remains stable. So, it's crucial for the theoretical research and clinical treatment to find the reason why antineoplastic miRNAs such as hsa-miR-142-3p are inactivated in the progression of HCC. The study on the interaction between miRNAs and lncRNAs will revolutionize our knowledge about cell structural network and regulatory network, and bring in immeasurable scientific and clinical value.

\section{Materials and methods \\ Cell culture}

Human hepatoma cell line, HepG2, obtained from the Cell Bank of the Chinese Academy of Sciences (Shanghai, China), were maintained in RPMI-1640 (Invitrogen, CA, USA) supplemented with 10\% Fetal bovine calf serum (FBS, Invitrogen, CA, USA). 293TN cells, purchased from ATCC (MD, USA), were maintained in Dulbecco minimum essential medium (DMEM, Invitrogen CA, USA) supplemented with 10\% FBS. All these adherent cells were passaged by $0.25 \%$ trypsin digestion (Invitrogen CA, USA) and incubated in an atmosphere of $5 \% \mathrm{CO}_{2}$ at $37^{\circ} \mathrm{C}$.

\section{Assessment of IncRNA-CR594175, hsa-miR142-3p, CTNNB1 protein and Wnt pathway related protein expression levels in HCC tumors and their metastasis}

Adjacent non-cancerous tissues, HCC, the metastatic from 24 patients (diagnosed in the First Affiliated Hospital of Zhengzhou University and detailed patient information was shown in Table 1) were collected, followed by total RNA extraction and Quantitative Real-time PCR (RT-qPCR) for measurement of lncRNA-CR594175 and hsa-miR142-3p level and total proteins were extracted and used for CTNNB1 and Wnt pathway related proteins (E-cadherin, C-myc, CyclinD1 and MMP-9) detection by western blotting.

\section{Lentivirus packaging}

A siRNA sequence complementarily binding to lncRNACR594175 was chosen. The target sequences of siRNA (5'-GAATCCTCGGAGACAGCAG-3') are homologous to lncRNA-CR594175.The oligonucleotide templates of these shRNAs were chemically synthesized and cloned into the linear pSIH1-H1-copGFP shRNA Vector (System Biosciences, CA, USA) which was obtained through digestion by BamH I and EcoR I (Takara, Dalian, China) and purification by agarose gel electrophoresis. An invalid siRNA sequence (5' - AATCGTCGAGGGCCAG ACA-3') was used as a negative control (NC). Sequencing was used to confirm the vectors constructed (pSIH1-shRNA-CR594175 and pSIH1-NC). The CDS sequence of human CTNNB1 (NM_001904.3) was 
Table 1 Clinicopathological features of 24 patients with metastatic HCC

\begin{tabular}{|c|c|c|c|c|}
\hline Number & Gender & Age & TNM stage & Metastasis Site \\
\hline 1 & $\mathrm{~F}$ & 55 & T2N1M1 & Lung \\
\hline 2 & M & 62 & T3N1M1 & Lung \\
\hline 3 & $F$ & 61 & T2NXM1 & Stomach \\
\hline 4 & $\mathrm{~F}$ & 55 & T2NoM1 & Head \\
\hline 5 & $\mathrm{~F}$ & 58 & T3NXM1 & Colon \\
\hline 6 & M & 66 & T3N0M1 & Lung \\
\hline 7 & M & 73 & T2N1M1 & Stomach \\
\hline 8 & $\mathrm{~F}$ & 55 & T3N1M1 & Colon \\
\hline 9 & M & 42 & T2N1M1 & Stomach \\
\hline 10 & $\mathrm{~F}$ & 58 & T3N0M1 & Lung \\
\hline 11 & M & 51 & T3N1M1 & Stomach \\
\hline 12 & M & 44 & T2NoM1 & Stomach \\
\hline 13 & $\mathrm{~F}$ & 53 & T3NXM1 & Lung \\
\hline 14 & $\mathrm{~F}$ & 66 & T3N1M1 & Colon \\
\hline 15 & M & 62 & T3N0M1 & Stomach \\
\hline 16 & M & 60 & T2NXM1 & Lung \\
\hline 17 & $\mathrm{~F}$ & 52 & T3N1M1 & Lung \\
\hline 18 & $\mathrm{~F}$ & 47 & T3N0M1 & Lung \\
\hline 19 & M & 52 & T2NoM1 & Bone \\
\hline 20 & $\mathrm{~F}$ & 58 & T2NoM1 & Stomach \\
\hline 21 & M & 69 & T3NXM1 & Lung \\
\hline 22 & $\mathrm{~F}$ & 70 & T2NXM1 & Lung \\
\hline 23 & $\mathrm{~F}$ & 42 & T4N1M1 & Colon \\
\hline 24 & M & 53 & T3N1M1 & Bone \\
\hline
\end{tabular}

$\mathrm{M}$, male; $\mathrm{F}$, female; TNM, tumor node metastasis.. Clinical stage category were ruled by 7 th edition of TNM

There are 24 pairs HCC tumors, their metastasis and adjacent normal tissues obtained from patients in the First Affiliated Hospital of Zhengzhou University between January 2013 and December 2017. Clinical stage category was ruled by 7 th edition of TNM

amplified by using the primers $5^{\prime}$-GGAATTCGCC ACCATGGCTACTCAAGCTGATTTG-3' and 5' CGGGATCC TTACAGGTCAGTATCAAACC-3', which contain an EcoRI cutting site and Kozak sequence and a BamhI cutting site, respectively, with the cDNA prepared by reverse transcription of RNA isolated from 293TN cells. The PCR product was digested and cloned into pcDH1-CMV lentiviral expressing vector; the recombinant vector was named pcDH1-CTNNB1. The products of the vectors were confirmed by DNA sequencing. Endotoxin free DNA was prepared in all cases.

One day before transfection, 293TN cells were seeded into 10-cm dishes (Corning, NY, USA). $2 \mu \mathrm{g}$ of each pSIH1-shRNA-CR594175 vector or pSIH1-NC and $10 \mu \mathrm{g}$ pPACK Packaging Plasmid Mix (System Biosciences) were co-transfected using Lipofectamine 2000 (Invitrogen) in accordance with the manufacturer's protocol. The medium was replaced with DMEM plus $1 \%$ FBS. Forty eight hours later, the supernatant was harvested and then cleared by centrifugation at $5000 \times \mathrm{g}$ at $4{ }^{\circ} \mathrm{C}$ for $5 \mathrm{~min}$, and passed through a $0.45 \mu \mathrm{m}$ PVDF membrane (Millipore, MI, USA). The titer of virus was determined by gradient dilution. The packaged lentiviruses were named as Lv-shRNA-CR594175 and Lv-NC. Recombinant lentivirus Lv-CTNNB1 and Lv-miR142-3p were packaged by following the same protocol.

\section{Genetic intervention through a lentiviral approach}

Cells were divided into four groups: a control group, LvNC group (infected with Lv-NC), Lv-shRNA-CR594175 group (infected with Lv-shRNA-CR594175) and LvCTNNB1 group (infected with Lv- CTNNB1). HepG2 in logarithmic phase growth were seeded to 6-well plates at $5 \times 10^{5}$ cells/well. One day later, viral solution was added at a multiplicity of infection (MOI) of 10 . The infection efficiency was evaluated by observing and analyzing the fluorescent mark $72 \mathrm{~h}$ after infection. Total RNA and protein were isolated from the cells and subjected to RT-qPCR and western blotting for lncRNA-CR594175 and CTNNB1 protein.

\section{Luciferase experiment}

Total RNA was extracted from HepG2, reversetranscribed into cDNA, and $2 \mu$ lof the reaction product subsequently used as a template for PCR. Primers targeting the $3^{\prime}$-UTR of the CTNNB1 gene were designed such that flanking $\mathrm{XbaI}$ restriction sites were introduced into the $127 \mathrm{bp}$ (base-pair) PCR product containing the 5'-AACACTA-3' hsa-miR-142-3p target site. The forward and reverse primer sequences were $5^{\prime}-$ GCTC TAGATTAAGAATTGAGTAATGG-3' and $5^{\prime}$-GCTC TAGA ACTAATTGGACCATTTTC-3', respectively. PCR reaction conditions were as follows: 35 cycles of a $94{ }^{\circ} \mathrm{C}$ denaturing step for $30 \mathrm{~s}$, a $55^{\circ} \mathrm{C}$ annealing step for $30 \mathrm{~s}$, and a $72^{\circ} \mathrm{C}$ elongation step for $10 \mathrm{~s}$. The PCR product was digested with $\mathrm{XbaI}$ (Takara) and cloned into the pGL3-promoter luciferase reporter vector (Promega, MI, USA) to generate the vector pGL3-wt-CTNNB1. The hsa-miR142-3p target site in the pGL3-WT-CTNNB1 vector was mutated from $5^{\prime}-$ AACACTA $-3^{\prime}$ to $5^{\prime}$ CATAACA $-3^{\prime}$ to construct the mutated reporter vector, pGL3-mt-CTNNB1. The products of all cloning and mutagenesis reactions were confirmed by DNA sequencing. Endotoxin free DNA was prepared in all cases. The hsa-miR142-3p mimic(5'-UGUAGUGUUUCCUACUUU AUGGAtt-3'), the hsa-miR142-3p inhibitor (5'UCCAUAAAGUAGGAAACACUACAtt-3), and negative control miRNA (NC,5'- UGUAGUGUUUCCUA CUUUAUGGAtt-3') were all chemically synthesized (Invitrogen). 
We used Targetscan (http://www.targetscan.org/) to predict whether a hsa-miR142-3p binding site exists within the 3 '-UTR of human CTNNB1 mRNA. The results showed that a seven-base hsa-miR142-3p seed sequence is present in the 3 '-UTR of CTNNB1 mRNA. The same tool was used to predict the binding sites of hsa-miR142-3p on lncRNA-CR594175.A suspension of 293TN cells in logarithmic phase growth was prepared and the number of viable cells counted using a hemocytometer in conjunction with trypan blue staining. The cells were seeded into six-well plates at a concentration of $2 \times 10^{5}$ cells per well and maintained in Dulbecco's Modified Eagle's medium supplemented with $10 \%$ FBS at $37{ }^{\circ} \mathrm{C}$ for $24 \mathrm{~h}$ in a $5 \% \mathrm{CO}_{2}$ atmosphere. The transfection of plasmid DNA and RNA was performed using Lipofectamine 2000 (Invitrogen). Transfection of cells with pRL-TK (100 ng) served as a reference for luciferase detection. Luciferase activity was measured using the dual luciferase reporter assay system (Promega) $48 \mathrm{~h}$ after transfection. The experiment to observe the effect of lncRNA-CR594175 depletion on the inhibition of luciferase by hsa-miR142-3p mimics was carried out in 293TN and HepG2 cells; the plasmid transfection and luciferase activity assay were the same as used in the validation of target sites of hsa-miR142-3p.

\section{Cellular proliferation assay}

HepG2 cells were divided into seven groups: a control group, Lv-NC group, Lv-shRNA-CR594175 group, LvmiR142-3p group, Lv-shRNA-CR594175 and Lv-miR1423p group, Lv-CTNNB1 group, and Lv-shRNA-CR594175 and Lv-CTNNB1 group. Fourty-eight hours after infection, HepG2 cells groups were trypsinized, and seeded into 96-well plates at a density of $1 \times 10^{4}$ cells per well. The cells were cultured under normal conditions and cell viability was examined using CCK-8 at 24-, 48-, and 72-h time points. Briefly, $10 \mathrm{uL}$ CCK- 8 solution (Dojindo, Japan) was added, and the cells then cultured under normal conditions for an additional $4 \mathrm{~h}$ before measurement of absorbance at $490 \mathrm{~nm}$.

\section{Cell invasion assay}

Cell invasion experiments were performed using the QCMTM 24-well Fluorimetric Cell Invasion Assay kit (Chemicon, International, MI, USA) according to the manufacturer's instructions. The kit uses an insert polycarbonate membrane with an $8-\mu \mathrm{m}$ pore size. The insert was coated with a thin layer of EC Matrix that occluded the membrane pores and blocked migration of noninvasive cells. Culture medium $(500 \mu \mathrm{l})$ supplemented with $10 \%$ FBS was used as chemoattractant. Cells that migrated and invaded the underside of the membrane were fixed in $4 \%$ paraformaldehyde. The invading cells were stained by Calcein-AM, and the number was then determined by fluorescence and reported as relative fluorescence units (RFUs).

\section{Effect of IncRNA-CR594175 silencing on the protein levels of CTNNB1, E-cadherin, C-myc, CyclinD1 and MMP-9}

HepG2 cells were divided into three groups: a control group, Lv-NC group and Lv-shRNA-CR594175. Cells in logarithmic phase were seeded to 6 -well plates at $5 \times 10^{5}$ cells/well. One day later, viral solution was added and the infection efficiency was evaluated by observing and analyzing the fluorescent mark $72 \mathrm{~h}$ after infection. Proteins were isolated and subjected to western blotting for CTNNB1, E-cadherin, C-myc, CyclinD1 and MMP-9 protein, respectively.

\section{RT-qPCR}

Total RNA was isolated with Trizol Reagent (Invitrogen) according to the manufacturer's instruction and reversely transcribed into cDNA using M-MLV Reverse Transcriptase (Takara, Japan) and oligo (dT)18 primer (Takara, Dalian, China). The following specific primers were used in RT-qPCR of human lncRNA-CR594175 and $\beta$-actin: IncRNA-CR594175: forward 5'-TTATGA CACATGCCACAACA-3' and reverse 5'-GGTACCTG TTATAAGTAGAATCA-3'; $\beta$-actin: forward5'-CCTG TACGCCAACACAGTGC-3' and reverse 5'-ATACTC CTGCTTGCTGATCC-3'. The lengths of amplified products were $109 \mathrm{bp}$ and $211 \mathrm{bp}$, respectively. RT-qPCR was performed using SYBR Premix Ex Taq kit and TP800 System (Takara). cDNA from $200 \mathrm{ng}$ total RNA was used as the template. The PCR reaction was carried out under the following conditions: 40 cycles of denaturation at $95^{\circ} \mathrm{C}$ for $10 \mathrm{~s}$, annealing at $60^{\circ} \mathrm{C}$ for $20 \mathrm{~s}$ and extension at $72{ }^{\circ} \mathrm{C}$ for $20 \mathrm{~s}$. The relative levels of mRNA and hsa-miR-142-3p were normalized using the $2^{-\Delta \mathrm{Ct}}$ method by using $\beta$-actin and U6 as the references. The PCR primers for hsa-miR142-3p or U6 were as follows: hsa-miR142-3p: forward: 5' - TGTAGTGTTTCCTACT TTATGGA-3' and reverse: 5'-GTCGTATCCAGTGC GTGTCGTG-3'; U6: forward:5'-GTGCTCGCTT CGGCAGCACAT-3' and reverse: 5'-TACCTTGCGA AGTGCTTAAAC-3'.

\section{Western blotting}

The total protein was extracted from the cells and tissues by using mammalian protein extraction reagent (Pierce, IL, USA). Equal amounts of protein (25 $\mu \mathrm{g}$ per lane) estimated by a bicinchoninic acid (BCA) protein assay kit (Pierce) were loaded onto (11\%) SDS-PAGE gels and transferred onto nitrocellulose membranes. The blots were probed with a monoclonal antibody against human CTNNB1 (1:400), E-cadherin (1:200), C-myc (1: 300), CyclinD1 (1:400), MMP-9 (1:250) and $\beta$-actin (1: 1000) (Santa Cruz, USA), followed by the secondary 
HRP-conjugated anti-mouse/rabbit antibody (Abcam, Cambridge, UK). After washing, the bands were detected by chemiluminescence and imaged with $\mathrm{X}$-ray films. $\beta$ actin was used as an endogenous reference for normalization.

\section{Animal xenografts}

Nude mice were purchased from Shanghai SLAC Laboratory Animal Co.,Ltd. (Shanghai, China) and housed at the animal experiment center of Zhengzhou University, where the implantation experiment was performed. All the protocols were previously approved by the Zhengzhou University Animal Ethics Committee. HepG2 cells $\left(1 \times 10^{6}\right)$ were suspended in $200 \mu \mathrm{l}$ medium, and injected subcutaneously into the flank regions of 48 female athymic nude mice. Two weeks after inoculation, visible subcutaneous tumors were detected, and the tumors were measured approximately $2.5 \mathrm{~mm}$ in diameter 3 weeks after inoculation. All animals were randomly divided into 3 groups (8 mice per group): the Model group, the NC group, and the lncRNA-CR594715silencing group. For the intervention groups, each animal received $30 \mu \mathrm{l}$ recombinant lentivirus $\left(1 \times 10^{8} \mathrm{IFU}\right)$ twice a week since the second week for 4 weeks, while the model group received the same volume of saline instead. Tumor diameter was measured weekly since the second week, and the data was used to plot the tumor growth curves. The formula for calculating the tumor volume was: $\mathrm{V}=1 / 2 \mathrm{a} \times \mathrm{b} 2$, $\mathrm{a}$ and $\mathrm{b}$ are the long and short diameters of the tumor, respectively.

\section{Statistical analysis}

All data are expressed as mean $\pm \mathrm{SD}$, and analyzed by one way ANOVA. Least Significant Difference (LSD) was used for multiple comparisons between any two means. $P$-values $<0.05$ were considered statistically significant. All statistical analysis was performed using SPSS 13.0 software.

\section{Results}

Assessment of mRNA and protein levels of CTNNB1 and hsa-miR-142-3p and IncRNA-CR594175 levels through RTqPCR and Western blotting in adjacent non-cancerous, primary HCC and metastatic tissues

The data of CTNNB1 mRNA and protein levels demonstrated that in comparison with adjacent noncancerous tissues, CTNNB1 protein was increased in HCC and its metastasis $(p<0.01)$, and more in HCC metastasis than in primary $\mathrm{HCC}(p<0.05)$; but there were no obvious differences between the mRNA levels in the three groups of tissues $(p>0.05)$. These results suggest that the high expression of CTNNB1 is due to inactivation of post-transcriptional regulation. The levels of lncRNA-CR594175 and hsa-miR142-3p in the adjacent tissues, $\mathrm{HCC}$ and its metastasis were positively correlated with CTNNB1 protein levels and was higher in the HCC and their metastasis than that in the adjacent tissues $(p<0.05)$ (Fig. 1a). We also evaluated the expression of downstream functional proteins of Wnt pathway, E-cadherin, C-myc, CyclinD1, and MMP-9, in HCC, in metastasis and adjacent tissue $(p<0.01)$, and we observed that protein levels were significantly higher in HCC metastasis than in primary HCC $(\mathrm{p}<0.05)$. A reverse trend was observed in E-cadherin to the three proteins mentioned above in these tissues (Fig. 1b).

\section{Effect of IncRNA-CR594175 silencing and CTNNB1 expression via lentiviral strategy on HCC cells}

Recombinant lentiviruses, Lv-NC, Lv-shRNACR594175 and Lv-CTNNB1, were used to infect HepG2. GFP (Green fluorescent protein) was detected in most of the cells $72 \mathrm{~h}$ after infection, and the proportion of GFP-expressing cells suggested that the gene delivery efficiency was higher than $95 \%$ in the HepG2 (Fig. 2a). LncRNA-CR594175 was significantly decreased by Lv-shRNA-CR594175 $(p<0.05)$, and no change in cells infected with Lv-CTNNB1 $(p>0.05)$ was obsserved; CTNNB1 protein level was significantly increased by Lv-CTNNB1 and decreased by Lv-shRNACR594175 $(p<0.05)$ (Fig. 2b). These findings suggest that lncRNA-CR594175 silencing down-regulated CTNNB1 expression in HepG2and that the overexpression of CTNNB1 had no obvious effect on lncRNA-CR594175.

\section{Luciferase experiments}

Our bioinformatics analysis identified a 7-base pair hsa-miR-142-3p in the 3' UTR of CTNNB1 mRNA. We therefore constructed luciferase reporter vectors to verify whether this site represents a valid hsamiR142-3p target. Reporter vectors containing the wild-type CTNNB1 3'-UTR or a variant were generated. The variant vector had the has-miR142-3p target site within the 3'-UTR of CTNNB1 mutated. Both reporter constructs expressed luciferase at a high level. However, the miR142-3p mimic significantly inhibited luciferase activity in cells transfected with the reporter vector encoding the wild type 3 '-UTR $(42.15 \pm 3.98$ vs. $8.07 \pm 0.88 ; p<0.01)$, while the miR142-3p inhibitor significantly increased luciferase activity in these cells $(42.15 \pm 3.98$ vs. $52.81 \pm 9.04 ; p<0.05)$ (Fig. 3a). Conversely, in cells transfected with the reporter vector encoding the mutated hsa-miR142-3p target site, neither the miR142-3p mimic nor the miR142-3p inhibitor had any significant effect on luciferase activity $(p>0.05)$. Co-transfection of miR142-3p-NC (non-targeting control) had no effect on the luciferase activity 


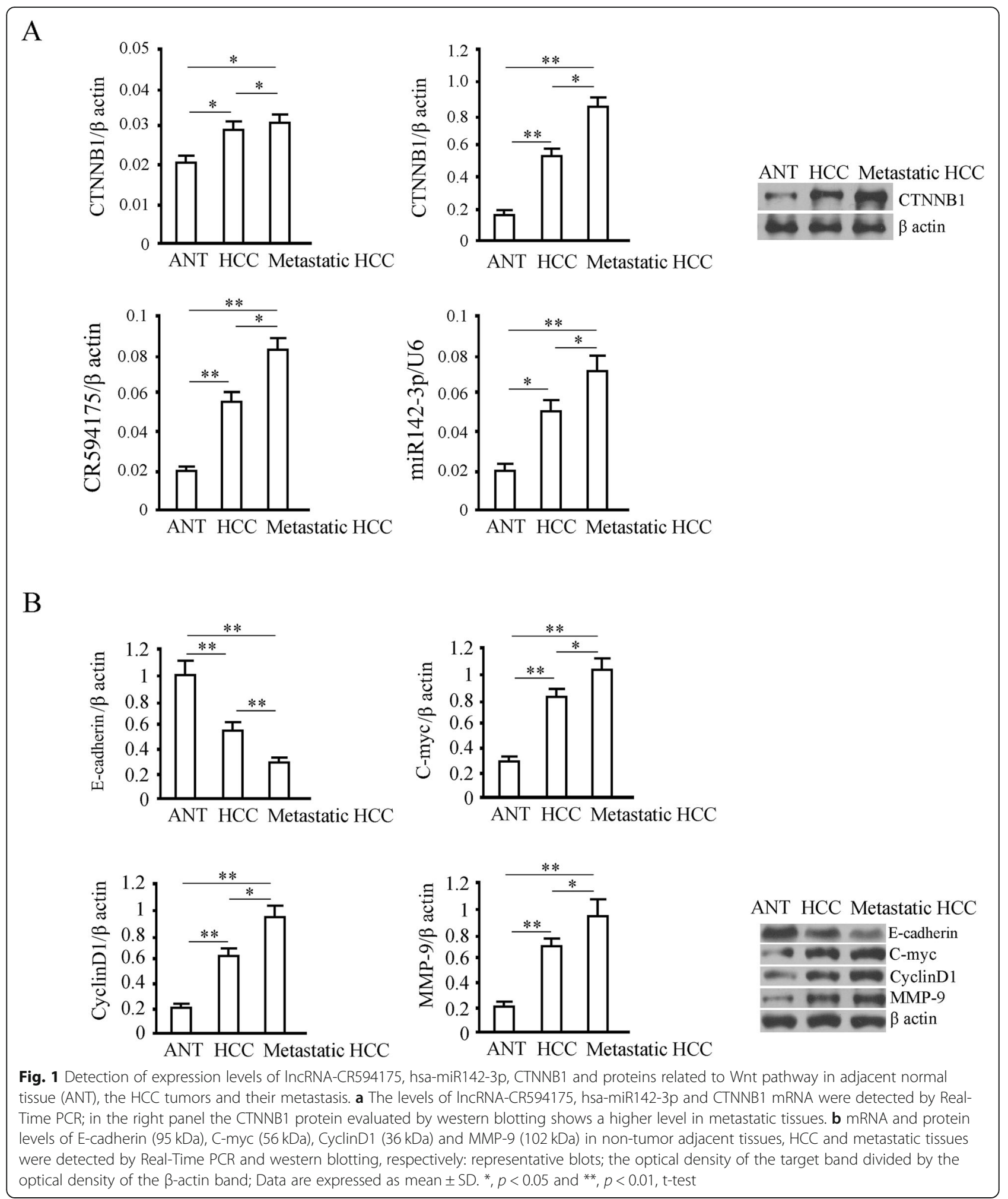

of either of the vectors $(p>0.05)$. These results verified the presence of a hsa-miR142-3p target site in the 3'-UTR of CTNNB1 mRNA and demonstrated that binding of hsa-miR142-3p to this target site down-regulated CTNNB1 expression (Fig. 3a). Interestingly, miR142-3p mimics lost its inhibition on the activity of luciferase expressed by wild-type (wt) luciferase reporter vector in HepG2, and regained the 
A

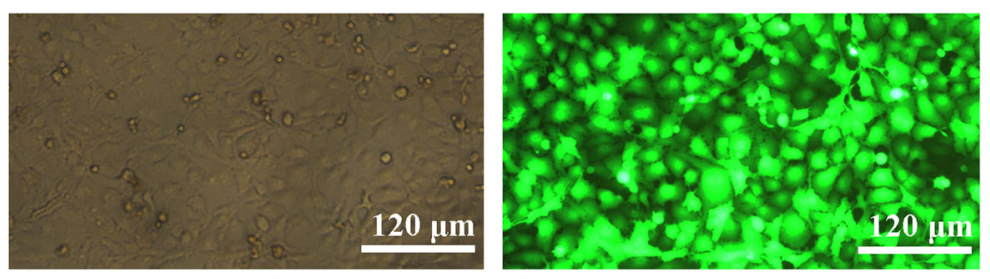

Cell infected with Lv-CR594175 (MOI=10) $72 \mathrm{hrs}$ later
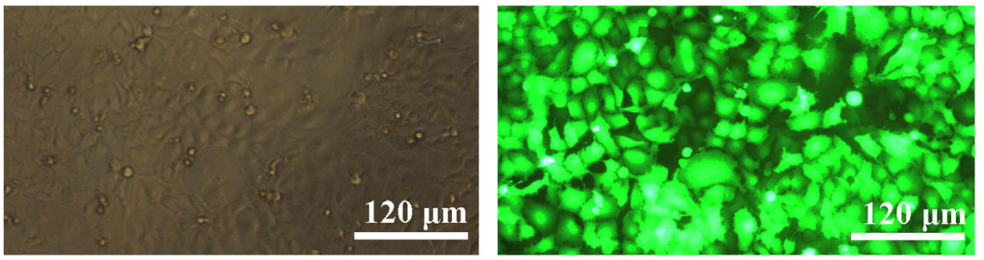

Cell infected with Lv-CTNNB1 (MOI=10) $72 \mathrm{hrs}$ later

$\mathrm{B}$
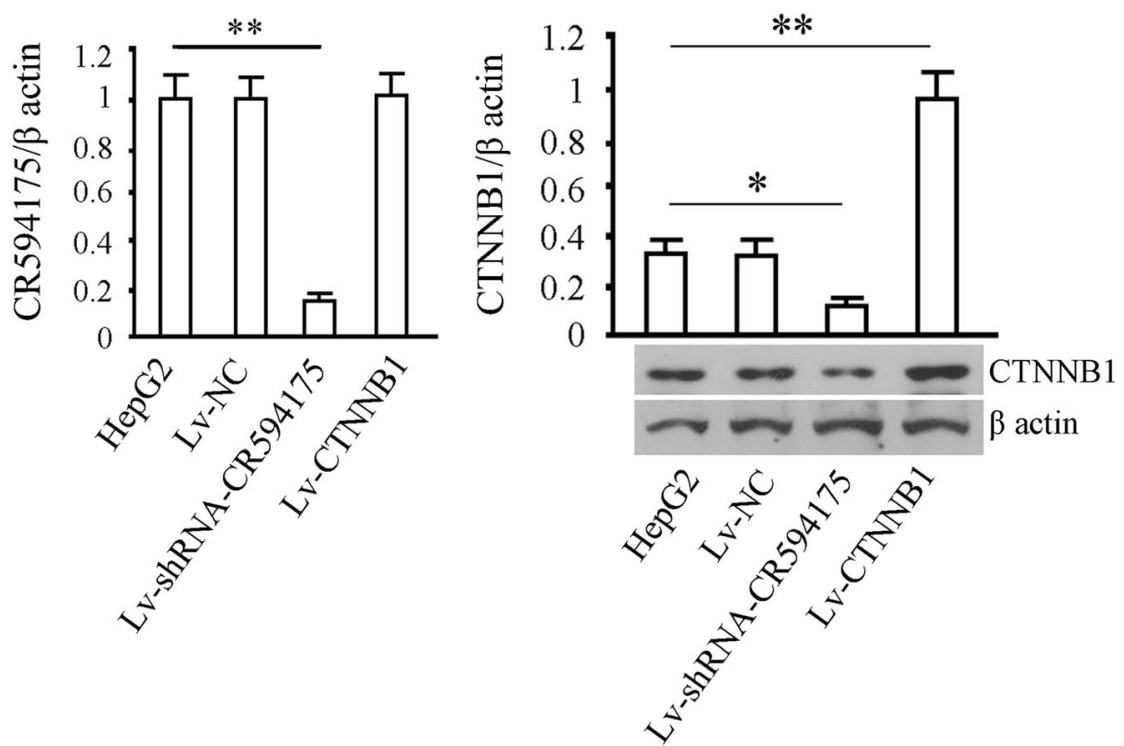

Fig. 2 Genetic intervention through a lentiviral approach. a GFP expression $72 \mathrm{~h}$ after HepG2 were infected with recombinant viruses Lv-shRNACR594175 and LV-CTNNB1. The infection rate was estimated by dividing the number of the cells expressing GFP with the number of all the cells in each view. For statistics, five views were randomly selected, and the mean was calculated; $\mathbf{b}$ Evaluation of IncRNA-CR594175 and CTNNB1 levels after infection with lentiviruses. For RT-qPCR and western blotting, the $\beta$ actin was used as internal reference. ${ }^{* *} p<0.01$, vs cell group. The tests were carried out on three biological triplicates, and data are expressed as the mean \pm SD

inhibition after lncRNA-CR594175 silencing (Fig. 3b). Taken together, these data suggested that lncRNACR594175 silencing could restore the negative regulation of hsa-miR-142-3p on its target gene CTNNB1 in HepG2 cells.

\section{Effect of IncRNA-CR594175 silencing on proliferation and invasion of HepG2 cells}

Cell proliferation assay showed that lncRNA-CR594175 silencing inhibited the proliferation of HepG2 cells $72 \mathrm{~h}$ after infection $(p<0.01$,vs. cell group, $72 \mathrm{~h})$, while CTNNB1 overexpression promoted the proliferation of HepG2 cells $(p<0.05$,vs. cell group, $72 \mathrm{~h})$, and could reverse the proliferation suppression caused by lncRNA-
CR594175 silencing ( $\mathrm{p}<0.05$,vs. IncRNA-CR594175 silencing group, $72 \mathrm{~h}$ ). Overexpression of miR142-3p showed a strong inhibitory activity of proliferation in the case of $\operatorname{lncRNA-CR594175} \operatorname{silenced}(p<0.01$,vs. cell group,72 h) (Fig. 4a). Therefore, it was suggested that lncRNA-CR594175 silencing inhibited cell proliferation by suppressing the expression of CTNNB1 protein. Compared with the control group, the invasion ability of HepG2 in the IncRNA-CR594175 silence group was significantly weakened ( $p<0.01$ vs. control group), significantly enhanced in CTNNB1 overexpression group and lncRNA-CR594175 silence combined CTNNB1 overexpression group ( $p<0.05$ vs. control group), and there was no significant change between the NC group or 
A
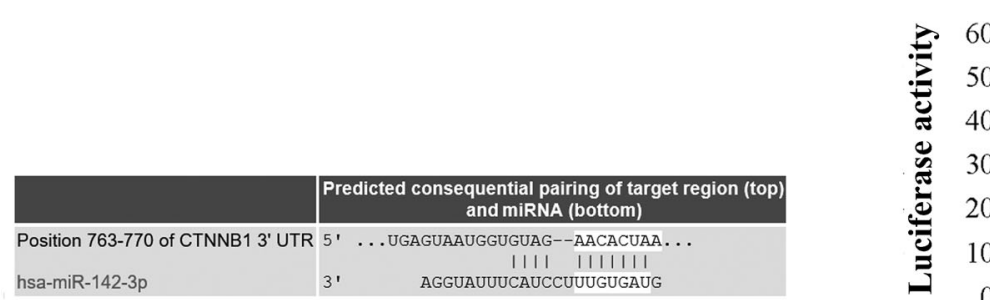

pGL3-wt-CTNNB1 pGL3-mt-CTNNB1 -
miR142-3p mimics miR142-3p inhibitor miR142-3p NC -

$\mathrm{B}$

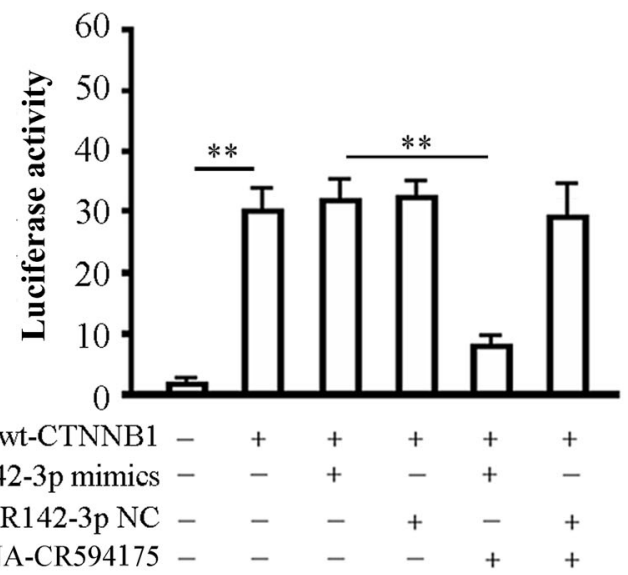
pSIH1-shRNA-CR594175 $\quad$ - $\quad-\quad \ldots++$
Seqence of CR594175

5 '-tggcatcatgattcacaagagccaaaaggtggaagcaatccaagtatccatc gatggataaatggataaacaaaatgtgagacatacatataattgaatattattc aacacta aaaaggaaggaaattatgacacatgccacaacatggatgaactttgaagacgttatgctaa atgataataacccagtctcgataaaaccaagaacggtatgattctacttataacaggtacct agagtggttgaatcctcggagacagcagaatggcacttgccagatgctgaggggaggga gaaacgtggagttactgtttaatggatacagattcagctttccaagatggaaagagtttgga atttaaatatgctt aacacta ctgaaccatacattttaaaatggetaagataggagatt-3'

aacacta $\rightarrow$ Binding sites of hsa-miR142-3p

Fig. 3 Hsa-miR142-3p binds to CTNNB1 3'UTR, which is interfered by IncRNA-CR594175. a 293TN cells were transfected with pGL3-wt-CTNNB1 or pGL3-mt-CTNNB1 in the presence or absence of miR142-3p-mimic or inhibitor and subjected to luciferase activity assay $48 \mathrm{~h}$ later. Left, predicted binding site of hsa-miR142-3p in 3'-UTR of CTNNB1; Right, effects of hsa-miR142-3p on the expression of a luciferase cassette encoding the CTNNB1 3'-UTR. The histogram shows the relative firefly luciferase activity for the different experimental groups. ${ }^{*}, p<0.05$, and ${ }^{* *}, p<0.01$, compared with the group transfected with the same vector but without the miR142-3p mimics or miR142-3p inhibitor. b Effect of IncRNACR594175 silencing on regulation of CTNNB1 by hsa-miR142-3p in HepG2 cells. Left, HepG2 cells were transfected with the indicated vectors and subjected to luciferase activity assay $48 \mathrm{~h}$ later. The histogram shows the relative firefly luciferase activity for the different experimental groups; right, prediction of the binding sites of hsa-miR-142-3p in IncRNA-CR594175. ${ }^{*}, p<0.05$, compared with the group transfected pGL3-wt-CTNNB1 and miR142-3p-mimics. Data are expressed as mean \pm SD of at least three independent experiments

miR142-3p overexpression group and control group $(p>$ 0.05 vs. control group). The invasive ability of HepG2 in the lncRNA-CR594175 silence group combined miR142$3 p$ overexpression group was significantly weakened than that of the control or NC group ( $p<0.01$ vs. control group or NC group), but there was no significant difference from the lncRNA-CR594175 silence group ( $p>0.05$ vs. miR142-3p overexpression group) (Fig. 4b). In vivo experiment showed that 4 consecutive weeks of treatment with Lv-shRNA-CR594175 significantly reduced the tumor volume. After administration for five weeks, the tumor volume of the model group was $701.21 \pm 54.13 \mathrm{~mm}^{3}$, the NC control group was $672.34 \pm$ $49.06 \mathrm{~mm}^{3}$ and the lncRNA-CR594175 silencing group was $212.31 \pm 57.71 \mathrm{~mm}^{3}$. The tumor inhibition rates in the NC group and lncRNA-CR594175 silenced group were 4.12 and $69.73 \%$, respectively, with a statistically significant difference between the lncRNA-CR594175 silenced group and the other two groups $(p<0.01$, vs. Model group or NC group) (Fig. 4c).

\section{Effect of IncRNA-CR594175 silencing on expression of downstream functional protein of Wnt pathway}

We assessed E-cadherin, C-myc, CyclinD1 and MMP-9 in the lncRNA-CR594175 silenced HepG2 cells. The results showed that $\mathrm{C}$-myc, CyclinD1 and MMP-9 were decreased and E-cadherin was increased by lncRNACR594175 silencing significantly $(\mathrm{p}<0.01)$ but no change in protein levels was observable after treatment with LvNC ( $>0.05$ ) (Fig. 5). The results indicated that lncRNACR594175 silencing could regulate the classic Wnt pathway by reducing CTNNB1 protein levels and the other Wnt pathway activation related proteins modulating cell proliferation and invasiveness (Fig. 5). 

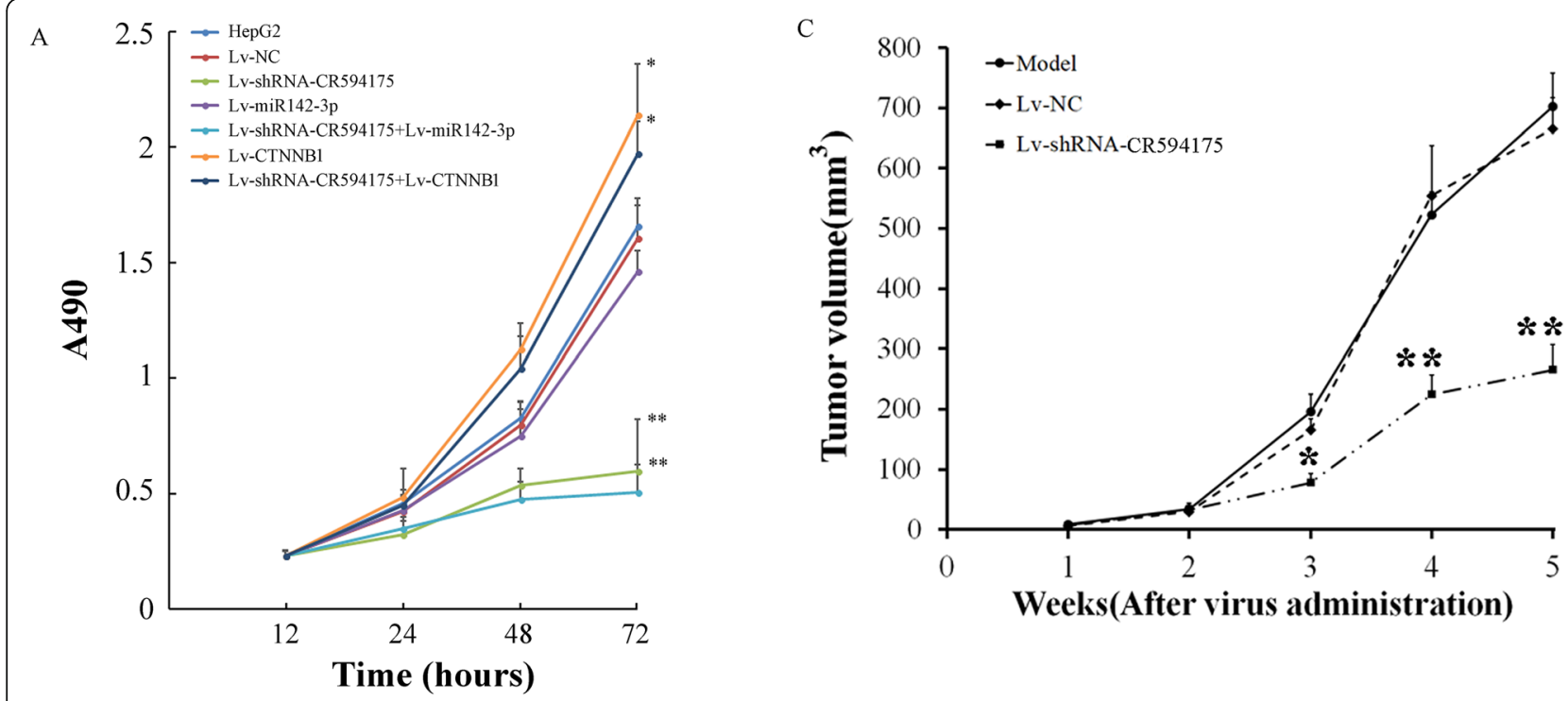

B
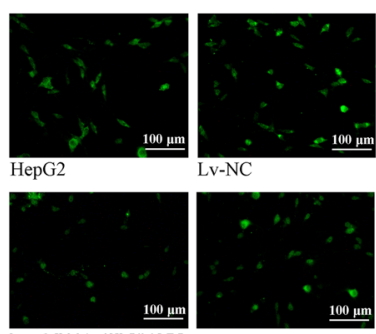
$\mathrm{Lv}-\mathrm{NC}$
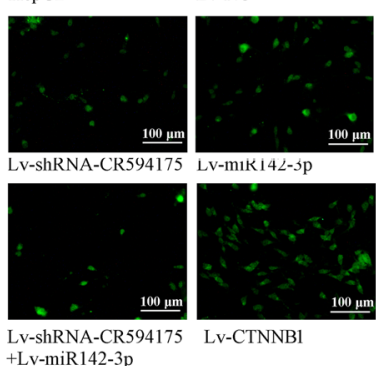

LV-miki4L-sp
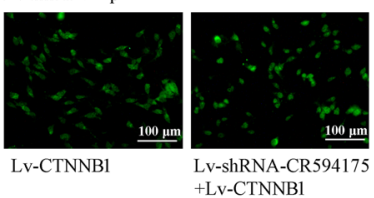

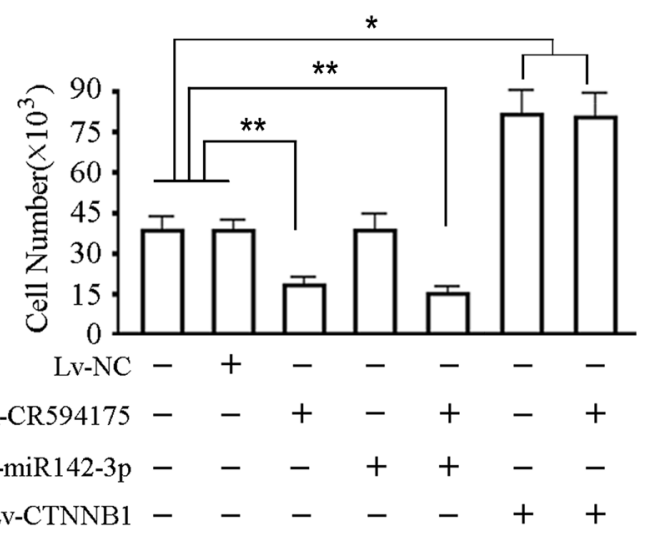

Fig. 4 Effects of IncRNA-CR594175 depletion on proliferation and invasion of HCC cells and In vivo tumor suppression. a Cell proliferation activity assay. HepG2 were infected with the indicated lentivirus and then seeded to 96-well plates and used for the detection of cell viability at the 0,24,48 and $72 \mathrm{~h}$. The $\mathrm{x}$-coordinate represents proliferation at different time points and the $\mathrm{y}$-coordinate represents the absorbance at $490 \mathrm{~nm}$. $\mathbf{b}$ Cell invasion assay: HepG2 cells $48 \mathrm{~h}$ after infection with the indicated lentiviruses; representative images of cells that seeded into the upper chamber of a transwell and passed through the basement membrane. c Growth curves of tumor in vivo. The $x$-coordinate represents the period of virus injection and the $y$-coordinate represents the tumor volume $\left(\mathrm{mm}^{3}\right)$. The formula for calculating the tumor volume $w a s: V=0.5 \times a \times b \times b$, where $\mathrm{a}$ and $\mathrm{b}$ are the long and short diameters of the tumor. The number of animals in one group was $12(n=12){ }^{* *} p<0.01,{ }^{*} p<0.05, \mathrm{t}$-test, data are expressed as the means \pm SD

\section{Discussion}

The invasion and metastasis of cancer refers to cancer cells that break away from the primary focus of the tumor and transfer to the neighbor where they proliferate into cancer of the same nature [13]. This process depends on the interaction between cancer cells and tumor microenvironment promoting their survival, growth, and angiogenesis, as well as invasion and metastasis [14]; therefore, inhibiting of proliferation and invasion of tumor cells is the key factor to inhibit tumor metastasis.
As an important type of regulators, IncRNAs exert their functions through a variety of ways. Although they were firstly regarded as by-products by RNA polymerase II, or transcriptional noise, recent studies have shown that IncRNAs are associated with multiple biological processes such as chromosome silencing, chromatin modification and transcriptional regulation $[15,16]$. The proportion of lncRNAs in the total transcripts of genome is far larger than that of encoding RNAs. And lncRNAs play crucial roles in the regulatory network by their interaction with DNA, RNA and proteins. In 

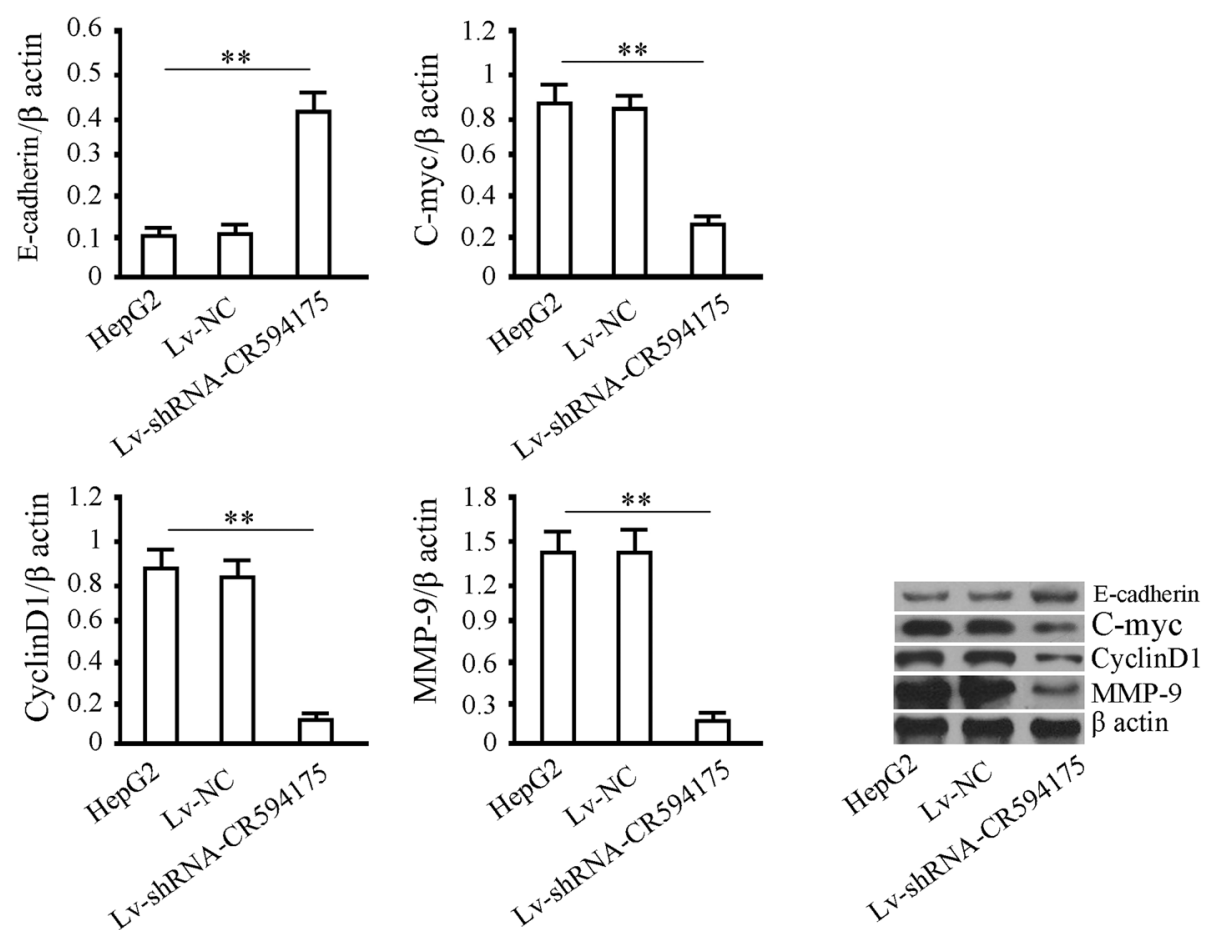

Fig. 5 Effects of CR594175 IncRNA-CR594175 silencing on E-cadherin, C-myc, CyclinD1 and MMP-9. HepG2 were infected with Lv-NC (negative control) or with Lv-shRNA-CR594175 and $72 \mathrm{~h}$ later were subjected to western blotting to test protein levels downstream of the WNT pathway. $\beta$ actin $\left(43 \mathrm{kDa}\right.$ ) was used as the loading control. Data are representative of at least three independent experiments. ${ }^{* *} p<0.01,{ }^{*} p<0.05 . t-t e s t$

addition to gene expression regulation, lncRNAs are closely related to evolution of species, embryonic development, metabolism and tumorigenesis. The evidence on involvement of lncRNAs in diseases including cancers will provide basis and target for diagnosis and treatment of diseases. Sun Shu-han et al. have found lncRNA-Dreh can inhibit hepatocellular carcinoma metastasis [17].

We screened for differential lncRNAs in several pairs of selected HCC and adjacent tissue by using lncRNA chips. The reason why lncRNA-CR594175 caught our attention was that its expression was not only increased in HCC than in adjacent non-cancerous tissues, but also was increased more in HCC metastases than in primary HCC, indicating that IncRNA-CR594175 would be associated with the process of $\mathrm{HCC}$ occurrence and metastasis. We knocked down IncRNA-CR594175 in HepG2 cells, and found that the proliferation and invasion were reduced, as well as downstream proteins of Wnt pathway cMyc, CyclinD1 and MMP-9, through Wnt pathway while E-cadherin shows an opposite behavior. So we believed that high lncRNA-CR594175 levels may contribute to metastases formation and tumor progression by regulating proteins downstream of Wnt pathway. Wnt signaling pathway is one of the key signaling pathways for cell proliferation and differentiation. To figure out how IncRNA-CR594175 promotes Wnt pathway in
$\mathrm{HCC}$, we analyzed key proteins involved in invasion and migration in HCC cells with IncRNA-CR594175 silencing and control cells, and found that the expression of CTNNB1 was consistent with lncRNA-CR594175, which was confirmed in primary HCC, metastatic HCC and adjacent tissue. Interestingly, our RIP (RNA Binding Protein immunoprecipitation) experiment showed that lncRNA-CR594175 did not bind to CTNNB1 directly (Data not shown). Next we quantified the transcription and protein levels of CTNNB1 in HCC with lncRNACR594175 silencing and found that CTNNB1 expression was abnormal at the post-transcription level, suggesting a mechanism of regulation of CTNNB1 expression following the high expression of lncRNA-CR594175. As a typical post-transcription regulating factor, miRNA naturally became our pointcut to investigate the relation between lncRNA-CR594175 and CTNNB1. Bioinformatics suggests that there is a 7 base-pair seeding region of hsa-miR142-3p on CTNNB1's 3'UTR and 2 seeding regions on the 600 base-pair lncRNA-CR594175. As a result, we speculated that elevated lncRNA-CR594175 bound to hsa-miR142-3p as miRNA sponge and disabled the negative regulation of CTNNB1 by hsa-miR142-3p, so CTNNB1 expression was increased and resulted in proliferation and invasion of HCC cells.

The interaction between IncRNAs and miRNAs has an important influence on the onset and development of 
cancer [18]. MiRNAs are able to regulate lncRNAs in a targeted way: a study has shown that miR-21 targets lncRNA GAS5 in addition to protein coding genes [19]. LncRNAs can also affect the onset and development of cancer by regulating expression of miRNAs [20]. According to existing studies, lncRNAs regulate miRNAs through three ways: (1) combining competitively to 3 'UTR of mRNAs so inhibiting negative regulation by miRNAs. Faghihi et al., for example, found that an antisense RNA can bind to BACE1 mRNA, competitively inhibiting the negative regulation of BACE1 by miRNA [21]; (2) to regulate target genes by forming premiRNAs after RNA splicing and producing specific miRNAs [22, 23]; and (3) to act as endogenous miRNA sponge to suppress miRNA function, so as to affect malignant biological behavior of cancer cells [24]. The most important finding of this study is that lncRNACR594175 silencing could restore the negative regulation of CTNNB1 by hsa-miR142-3p to inhibit cancer, directly based on following facts: (1) hsa-miR142-3p negatively regulated CTNNB1 by binding to its 3'UTR, which was found in HCC with lncRNA-CR594175 silencing but not those with high lncRNA-CR594175 expression levels; (2) LncRNA-CR594175 silencing inhibited proliferation and invasion of HCC cells, which was reversed by overexpression of CTNNB1; (3) Overexpression of hsamiR142-3p had no observable effect on proliferation and invasion of HCC cells, but inhibited proliferation and invasion of HCC cells when lncRNA-CR594175 was depleted. Considering that CTNNB1 overexpressed by the lentiviral system has no wild 3'UTR, it would not be affected by miRNA. So we think that there is a lncRNACR594175/hsa-miR-142-3p/CTNNB1 axis regulating metastasis formation in HCC.

\section{Conclusion}

The study demonstrates that lncRNA-CR594175 plays a key role in the process of HCC metastasis, and offers a possible explanation about why hsa-miR142-3p loses its basic function of resisting HCC tumors. In the long run, lncRNAs will not only be a direct target for gene therapy but it can also be used together with miRNAs for a better effect.

\section{Abbreviations}

HCC: Hepatocellular carcinoma; CTNNB1: Catenin, beta-1; UTR: Untranslated Regions; FBS: Fetal bovine serum; DMEM: Dulbecco minimum essential medium; NC: Negative control; bp: base-pair; LSD: Least Significant Difference; GFP: Green fluorescent protein

\section{Acknowledgements}

Not applicable.

\section{Authors' contributions}

LQ and YXX performed the data curation, methodology, project administration and wrote original draft. YMJ, LXK, ZXJ, LYJ, CZ, FQX, LWC collected data and performed statistical analysis. SLJ and LWC reviewed and edited the manuscript. All authors read and approved the final manuscript.
Funding

Natural Science Foundation of Henan Province.

\section{Availability of data and materials}

All data generated or analyzed during this study are included in this published article [and its supplementary information files].

Ethics approval and consent to participate

Not applicable.

\section{Consent for publication}

Not applicable.

\section{Competing interests}

The authors declare that they have no competing interests.

\section{Author details}

${ }^{1}$ Department of Emergency, The Fifth Affiliated Hospital of Zhengzhou University, Zhengzhou 450052, China. ²Department of Oncology, The First Affiliated Hospital of Zhengzhou University, Zhengzhou 450052, China. ${ }^{3}$ Department of Pathology, The First Affiliated Hospital of Zhengzhou University, Zhengzhou 450052, China.

Received: 26 February 2020 Accepted: 17 September 2020

Published online: 22 September 2020

\section{References}

1. Mulcahy MF. Management of hepatocellular cancer [J]. Curr Treat Options in Oncol. 2005:6(5):423-35.

2. Bosch FX, Ribes J, Diaz M, et al. Primary liver cancer: worldwide incidence and trends [J]. Gastroenterology. 2004;127(5 Suppl 1):S5-S16.

3. El-Serag HB. Hepatocellular carcinoma [J]. N Engl J Med. 2011;365(12):111827.

4. Yang $H$, Lin $M$, Xiong $F$, et al. Combined lysosomal protein transmembrane 4 beta-35 and argininosuccinate synthetase expression predicts clinical outcome in hepatocellular carcinoma patients [J]. Surg Today. 2011;41(6): 810-7.

5. Abdelmohsen K, Gorospe M. Noncoding RNA control of cellular senescence [J]. Wiley Interdiscip Rev RNA. 2015;6(6):615-29.

6. Alaei-Mahabadi B, Larsson E. Limited evidence for evolutionarily conserved targeting of long non-coding RNAs by microRNAs [J]. Silence. 2013;4(1):4.

7. Schmitt AM, Chang HY. Long noncoding RNAs in Cancer pathways [J]. Cancer Cell. 2016;29(4):452-63.

8. Li $\mathrm{X}$, Zhu $\mathrm{Y}, \mathrm{He} \mathrm{H}$, et al. Synergistically killing activity of aspirin and histone deacetylase inhibitor valproic acid (VPA) on hepatocellular cancer cells [J]. Biochem Biophys Res Commun. 2013:436(2):259-64.

9. Behari J, Zeng G, Otruba W, et al. R-Etodolac decreases beta-catenin levels along with survival and proliferation of hepatoma cells [J]. J Hepatol. 2007; 46(5):849-57.

10. Yang $C, X u$ Y, Cheng F, et al. miR-1301 inhibits hepatocellular carcinoma cell migration, invasion, and angiogenesis by decreasing Wnt/beta-catenin signaling through targeting BCL9[J]. Cell Death Dis. 2017;8(8):e2999.

11. Jin Y, Wang J, Han J, et al. MiR-122 inhibits epithelial-mesenchymal transition in hepatocellular carcinoma by targeting Snail1 and Snail2 and suppressing WNT/beta-cadherin signaling pathway [J]. Exp Cell Res. 2017; 360(2):210-7.

12. Ashmawy AM, Elgeshy KM, Abdel SE, et al. Crosstalk between liver-related microRNAs and Wnt/beta-catenin pathway in hepatocellular carcinoma patients [J]. Arab J Gastroenterol. 2017;18(3):144-50.

13. Fidler IJ. Tumor heterogeneity and the biology of cancer invasion and metastasis [J]. Cancer Res. 1978;38(9):2651-60.

14. Salvatore $\mathrm{V}$, Teti $\mathrm{G}$, Focaroli S, et al. The tumor microenvironment promotes cancer progression and cell migration [J]. Oncotarget. 2017;8(6):9608-16.

15. Bergmann $\mathrm{JH}$, Spector DL. Long non-coding RNAs: modulators of nuclear structure and function [J]. Curr Opin Cell Biol. 2014;26:10-8.

16. Yamashita A, Shichino $Y$, Yamamoto $M$. The long non-coding RNA world in yeasts [J]. Biochim Biophys Acta. 2016;1859(1):147-54.

17. Huang JF, Guo YJ, Zhao CX, et al. Hepatitis B virus X protein (HBx)-related long noncoding RNA (IncRNA) down-regulated expression by HBX (Dreh) inhibits hepatocellular carcinoma metastasis by targeting the intermediate filament protein vimentin [J]. Hepatology. 2013;57(5):1882-92. 
18. Tam C, Wong JH, Tsui S, et al. LncRNAs with miRNAs in regulation of gastric, liver, and colorectal cancers: updates in recent years [J]. Appl Microbiol Biotechnol. 2019;103(12):4649-77.

19. Pickard MR, Williams GT. Molecular and cellular mechanisms of action of tumour suppressor GAS5 LncRNA []]. Genes (Basel). 2015;6(3):484-99.

20. Gutschner T, Diederichs $\mathrm{S}$. The hallmarks of cancer: a long non-coding RNA point of view [J]. RNA Biol. 2012;9(6):703-19.

21. Faghihi MA, Modarresi F, Khalil AM, et al. Expression of a noncoding RNA is elevated in Alzheimer's disease and drives rapid feed-forward regulation of beta-secretase []]. Nat Med. 2008;14(7):723-30.

22. Keniry A, Kyba M, Dandolo L, et al. The H19 lincRNA is a developmental reservoir of miR-675 that suppresses growth and Igf1r.[J]. Nat Cell Biol. 2012; 14(7):659-65.

23. Wilusz JE, Sunwoo H, Spector DL. Long noncoding RNAs: functional surprises from the RNA world [J]. Genes Dev. 2009;23(13):1494-504.

24. Brodersen P, Sakvarelidze-Achard L, Schaller H, et al. Isoprenoid biosynthesis is required for miRNA function and affects membrane association of ARGONAUTE 1 in Arabidopsis [J]. Proc Natl Acad Sci U S A. 2012;109(5): 1778-83.

\section{Publisher's Note}

Springer Nature remains neutral with regard to jurisdictional claims in published maps and institutional affiliations.

Ready to submit your research? Choose BMC and benefit from:

- fast, convenient online submission

- thorough peer review by experienced researchers in your field

- rapid publication on acceptance

- support for research data, including large and complex data types

- gold Open Access which fosters wider collaboration and increased citations

- maximum visibility for your research: over $100 \mathrm{M}$ website views per year

At $\mathrm{BMC}$, research is always in progress.

Learn more biomedcentral.com/submissions 